DESY 10-099

arXiv:1007.0206 [hep-ph]

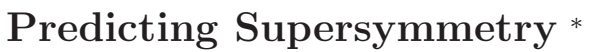

\author{
S. Heinemeyer讨 AND G. Weiglein讯 \\ ${ }^{1}$ Instituto de Física de Cantabria (CSIC-UC), Santander, Spain \\ ${ }^{2}$ DESY, Notkestraße 85, D-22603 Hamburg, Germany
}

\begin{abstract}
We review the result of SUSY parameter fits based on frequentist analyses of experimental constraints from electroweak precision data, $(g-2)_{\mu}, B$ physics and cosmological data. We investigate the parameters of the constrained MSSM (CMSSM) with universal soft supersymmetry-breaking mass parameters, and a model with common non-universal Higgs mass parameters in the superpotential (NUHM1). Shown are the results for the SUSY and Higgs spectrum of the models. Many sparticle masses are highly correlated in both the CMSSM and NUHM1, and parts of the regions preferred at the $68 \%$ C.L. are accessible to early LHC running. The best-fit points could be tested even with $1 \mathrm{fb}^{-1}$ at $\sqrt{s}=7 \mathrm{TeV}$.
\end{abstract}

\footnotetext{
*invited talk given by S.H. at the Loops \& Legs 2010, April 2010, Wörlitz, Germany

$\dagger$ email: Sven.Heinemeyer@cern.ch

‡email: Georg.Weiglein@desy.de
} 


\title{
Predicting Supersymmetry
}

\author{
S. Heinemeyer ${ }^{\mathrm{a}}$, G. Weiglein ${ }^{\mathrm{b}}$ \\ anstituto de Física de Cantabria (CSIC-UC), E-39005 Santander, Spain \\ bDESY, Notkestrasse 85, D-22603 Hamburg, Germany
}

We review the result of SUSY parameter fits based on frequentist analyses of experimental constraints from electroweak precision data, $(g-2)_{\mu}, B$ physics and cosmological data. We investigate the parameters of the constrained MSSM (CMSSM) with universal soft supersymmetry-breaking mass parameters, and a model with common non-universal Higgs mass parameters in the superpotential (NUHM1). Shown are the results for the SUSY and Higgs spectrum of the models. Many sparticle masses are highly correlated in both the CMSSM and NUHM1, and parts of the regions preferred at the $68 \%$ C.L. are accessible to early LHC running. The best-fit points could be tested even with $1 \mathrm{fb}^{-1}$ at $\sqrt{s}=7 \mathrm{TeV}$.

\section{INTRODUCTION}

Supersymmetry (SUSY) is one of the favored ideas for physics beyond the Standard Model (SM) that may soon be explored at the Large Hadron Collider (LHC). Here we review the results from frequentist analyses [1,2] of the parameter spaces of the constrained minimal supersymmetric extension of the Standard Model (CMSSM) - in which the soft SUSY-breaking scalar and gaugino masses are each constrained to universal values $m_{0}$ and $m_{1 / 2}$, respectively - and the NUHM1 - in which the soft SUSY-breaking contributions to the Higgs masses are allowed to have a different but common value. Both models have a common trilinear coupling $A_{0}$ at the GUT scale and $\tan \beta$ (the ratio of the two vacuum expectation values) as a low-energy input. An overview about the existing literature on this subject can be found in Ref. [1].

The fit includes precision electroweak data, the anomalous magnetic moment of the muon, $(g-2)_{\mu}, B$-physics observables (such as rates for $\mathrm{BR}(b \rightarrow s \gamma)$ and $\operatorname{BR}\left(B_{u} \rightarrow \tau \nu_{\tau}\right)$, and the upper limit on $\left.\operatorname{BR}\left(B_{s} \rightarrow \mu^{+} \mu^{-}\right)\right), K$-physics observables, the bound on the lightest MSSM Higgs boson mass, $M_{h}$, and the cold dark matter (CDM) density inferred from astrophysical and cosmolog- ical data 1 , assuming that this is dominated by the relic density of the lightest neutralino, $\Omega_{\chi} h^{2}$.

\section{THE MASTERCODE}

We define a global $\chi^{2}$ likelihood function, which combines all theoretical predictions with experimental constraints:

$$
\begin{aligned}
\chi^{2} & =\sum_{i}^{N} \frac{\left(C_{i}-P_{i}\right)^{2}}{\sigma\left(C_{i}\right)^{2}+\sigma\left(P_{i}\right)^{2}} \\
& +\chi^{2}\left(M_{h}\right)+\chi^{2}\left(\mathrm{BR}\left(B_{s} \rightarrow \mu \mu\right)\right) \\
& +\chi^{2}(\mathrm{SUSY} \text { search limits }) \\
& +\sum_{i}^{M} \frac{\left(f_{\mathrm{SM}_{i}}^{\mathrm{obs}}-f_{\mathrm{SM}_{i}}\right)^{\mathrm{fit}}}{\sigma\left(f_{\mathrm{SM}_{i}}\right)^{2}} .
\end{aligned}
$$

Here $N$ is the number of observables studied, $C_{i}$ represents an experimentally measured value (constraint), and each $P_{i}$ defines a prediction for the corresponding constraint that depends on the supersymmetric parameters. The experimental uncertainty, $\sigma\left(C_{i}\right)$, of each measurement is taken to be both statistically and systematically independent of the corresponding theoreti-

\footnotetext{
${ }^{1}$ We did not include the constraint imposed by the experimental upper limit on the spin-independent DM scattering cross section $\sigma_{p}^{\mathrm{SI}}$, which is subject to astrophysical and hadronic uncertainties.
} 
cal uncertainty, $\sigma\left(P_{i}\right)$, in its prediction. We denote by $\chi^{2}\left(M_{h}\right)$ (see below) and $\chi^{2}\left(\operatorname{BR}\left(B_{s} \rightarrow\right.\right.$ $\mu \mu)$ ) the $\chi^{2}$ contributions from two measurements for which only one-sided bounds are available so far. Similarly, we include the lower limits from the direct searches for SUSY particles at LEP [3] as one-sided limits, denoted by " $\chi^{2}$ (SUSY search limits)" in Eq. (1).

In order to derive a reliable theoretical prediction of an observable (denoted as $P_{i}$ in Eq. (1)) and to obtain a correspondingly small theory (intrinsic) uncertainty (denoted as $\sigma\left(P_{i}\right)$ in Eq. (10) it is crucial to have higher-order corrections (beyond the one-loop level) at hand. As an example, including SM corrections up to four-loop and MSSM corrections up to the two-loop level, the theory uncertainties of the $W$ boson mass, $M_{W}$, and the effective leptonic weak mixing angle, $\sin ^{2} \theta_{\text {eff }}$ are now at the level of (depending in the MSSM on the SUSY mass scale)

$$
\begin{aligned}
\delta M_{W}^{\mathrm{intr}, \mathrm{SM}} & =4 \mathrm{MeV}[4], \\
\delta M_{W}^{\mathrm{intr}, \mathrm{MSSM}} & =5-10 \mathrm{MeV}[5], \\
\delta \sin ^{2} \theta_{\mathrm{eff}}^{\mathrm{int}, \mathrm{SM}} & =4.7 \times 10^{-5}[6], \\
\delta \sin ^{2} \theta_{\mathrm{eff}}^{\mathrm{intr}, \mathrm{MSSM}} & =(4.9-7.1) \times 10^{-5}[7] .
\end{aligned}
$$

Other examples are $\operatorname{BR}(b \rightarrow s \gamma)$, where in the SM the intrinsic uncertainty is now at the level of $\sim 0.27 \times 10^{-4}$ [ ] (adding errors in quadrature) or $(g-2)_{\mu}$ with a theory uncertainty of less than $4.9 \times 10^{-10}$ 9]. Many calculations and tools used in our analyses were presented at "Loops \& Legs" conferences over the past decade, see Ref. [10].

The main computer code for our evaluations is the MasterCode [1, 2, 11, 13, which includes the following theoretical codes. For the RGE running of the soft SUSY-breaking parameters, it uses SoftSUSY [14], which is combined consistently with the codes used for the various low-energy observables: FeynHiggs [15 18 is used for the evaluation of the Higgs masses and $a_{\mu}^{\text {SUSY }}$ (see also 19,20]), for the other electroweak precision data we have included a code based on [5, 7], SuFla [21,22] and SuperIso [23,24] are used for flavor-related observables, and for darkmatter-related observables MicrOMEGAs [25] and DarkSUSY [26] are included. In the combination of the various codes, MasterCode makes extensive use of the SUSY Les Houches Accord [27,28].

The experimental constraints used in our analyses are listed in Table 1 of Ref. [1. One important comment concerns our implementation of the LEP constraint on $M_{h}$. The value quoted in the Table 1 of Ref. [1, $M_{H}>114.4 \mathrm{GeV}$, was derived within the SM 29], and is applicable to the CMSSM, in which the relevant Higgs couplings are very similar to those in the SM [30,31, so that the SM exclusion results can be used, supplemented with an additional theoretical uncertainty: we evaluate the $\chi^{2}\left(M_{h}\right)$ contribution within the CMSSM using the formula

$\chi^{2}\left(M_{h}\right)=\frac{\left(M_{h}-M_{h}^{\text {limit }}\right)^{2}}{(1.1 \mathrm{GeV})^{2}+(1.5 \mathrm{GeV})^{2}}$,

with $M_{h}^{\text {limit }}=115.0 \mathrm{GeV}$ for $M_{h}<115.0 \mathrm{GeV}$. Larger masses do not receive a $\chi^{2}\left(M_{h}\right)$ contribution. We use $115.0 \mathrm{GeV}$ so as to take into account experimental systematic effects. The $1.5 \mathrm{GeV}$ in the denominator corresponds to a convolution of the likelihood function with a Gaussian function, $\tilde{\Phi}_{1.5}(x)$, normalized to unity and centered around $M_{h}$, whose width is $1.5 \mathrm{GeV}$, representing the theory uncertainty on $M_{h}$ [17. In this way, a theoretical uncertainty of up to $3 \mathrm{GeV}$ is assigned for $\sim 95 \%$ of all $M_{h}$ values corresponding to one CMSSM parameter point. The $1.1 \mathrm{GeV}$ term in the denominator corresponds to a parameterization of the $C L_{s}$ curve given in the final SM LEP Higgs result [29]. Within the NUHM1 the situation is somewhat more involved, since, for instance, a strong suppression of the $Z Z h$ coupling can occur, invalidating the SM exclusion bounds. In order to find a more reliable $95 \%$ C.L. exclusion limit for $M_{h}$ in the case that the SM limit cannot be applied, we use a specially adopted procedure outlined in Ref. 1 (using the results of the code HiggsBounds 32 that incorporates the LEP [33] (and Tevatron) limits on neutral Higgs boson searches).

Our statistical treatment of the CMSSM and NUHM1 makes use of a large sample of points (about $2.5 \times 10^{7}$ ) in the SUSY parameter spaces obtained with the Markov Chain Monte Carlo (MCMC) technique. Our analysis is entirely fre- 

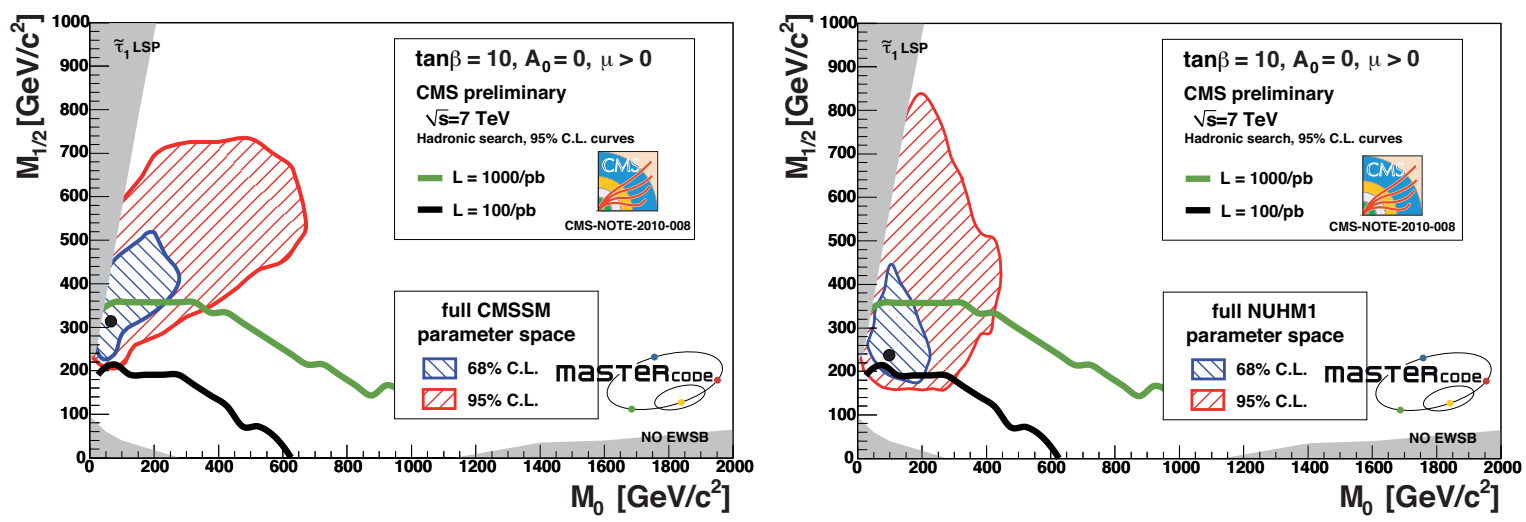

Figure 1. The $\left(m_{0}, m_{1 / 2}\right)$ plane in the CMSSM (left) and the NUHM1 (right). The dark shaded area at low $m_{0}$ and high $m_{1 / 2}$ is excluded due to a scalar tau LSP, the light shaded areas at low $m_{1 / 2}$ do not exhibit electroweak symmetry breaking. Shown in both plots are the best-fit point, indicated by a filled circle, and the 68 (95)\% C.L. contours from our fit as dark grey/blue (light grey/red) overlays, scanned over all $\tan \beta$ and $A_{0}$ values. The $95 \%$ C.L. exclusion curves (hadronic search channel) at CMS with $1(0.1) \mathrm{fb}^{-1}$ at $7 \mathrm{TeV}$ center-of-mass energy is shown as green/light gray (black) solid curve.

quentist, and avoids any ambiguity associated with the choices of Bayesian priors.

\section{PREDICTIONS FOR SPARTICLE MASSES}

For the parameters of the best-fit CMSSM point we find $m_{0}=60 \mathrm{GeV}, m_{1 / 2}=310 \mathrm{GeV}$, $A_{0}=130 \mathrm{GeV}, \tan \beta=11$ and $\mu=400 \mathrm{GeV}$, yielding the overall $\chi^{2} / \mathrm{N}_{\text {dof }}=20.6 / 19(36 \%$ probability) and nominally $M_{h}=114.2 \mathrm{GeV}$. The corresponding parameters of the best-fit NUHM1 point are $m_{0}=150 \mathrm{GeV}, m_{1 / 2}=$ $270 \mathrm{GeV}, A_{0}=-1300 \mathrm{GeV}, \tan \beta=11$ and $m_{h_{1}}^{2}=m_{h_{2}}^{2}=-1.2 \times 10^{6} \mathrm{GeV}^{2}$ or, equivalently, $\mu=1140 \mathrm{GeV}$, yielding $\chi^{2}=18.4$ (corresponding to a similar fit probability as in the CMSSM) and $M_{h}=120.7 \mathrm{GeV}$.

In Fig. 1] we display the best-fit value and the $68 \%$ and $95 \%$ likelihood contours for the CMSSM (left plot) and the NUHM1 (right plot) in the $\left(m_{0}, m_{1 / 2}\right)$ plane, obtained as described above from a fit taking into account all experimental constraints. We also show exclusion contours for the hadronic search mode (jets plus missing energy) at CMS. The green (light gray) solid line shows the $95 \%$ C.L. exclusion contour for CMS for $1 \mathrm{fb}^{-1}$ at $\sqrt{s}=7 \mathrm{TeV} 34$. The black solid line shows the corresponding results for only $0.1 \mathrm{fb}^{-1}$. (Similar results hold for ATLAS.) One can see that with $1 \mathrm{fb}^{-1}$ the best-fit points can be tested, together with a sizable part of the whole $68 \%$ C.L. preferred regions. In the case of the NUHM1 (right plot) nearly the $68 \%$ C.L. region could be probed.

We now review the results for the predictions of sparticles masses in the CMSSM and the NUHM1, which are summarized in Fig. 2. The results for the CMSSM spectrum are shown in the left plot, and for the NUHM1 in the right plot. We start our discussion with the gluino mass, $m_{\tilde{g}}$. In both the CMSSM and the NUHM1, the best-fit points have relatively low values of $m_{\tilde{g}} \sim 750$ and $\sim 600 \mathrm{GeV}$, respectively. These favored values are well within the range even of the early operations of the LHC with reduced centre-of-mass energy and limited luminosity, in accordance with the result of Fig. 1. However, even quite large values of $m_{\tilde{g}} \lesssim 2.5 \mathrm{TeV}$ are allowed at the 3 $\sigma\left(\Delta \chi^{2}=9\right.$ ) level (not shown in Fig. 2). The LHC should be able to discover a gluino with $m_{\tilde{g}} \sim 2.5 \mathrm{TeV}$ with $100 / \mathrm{fb}$ of integrated lumi- 

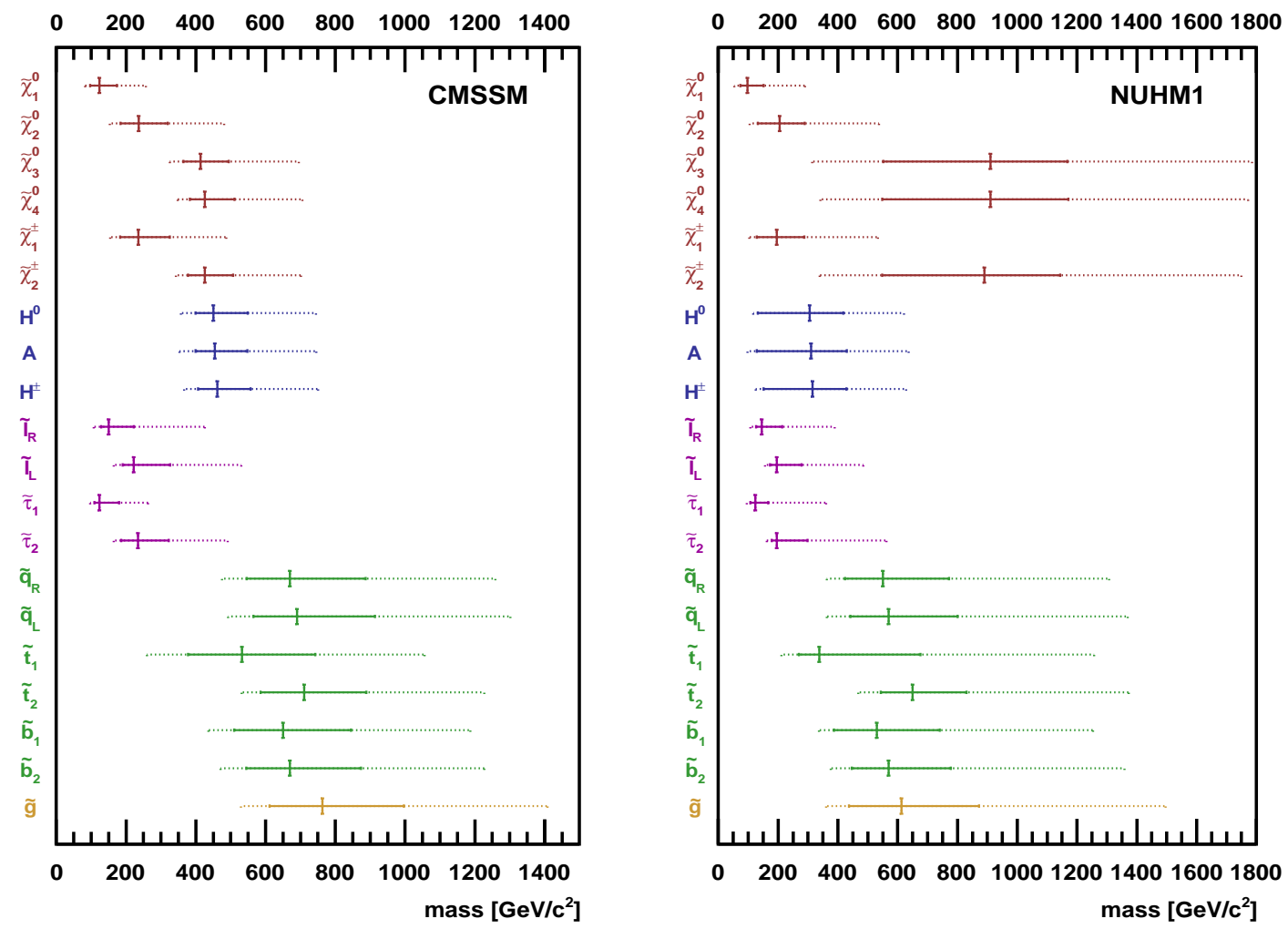

Figure 2. Spectra in the CMSSM (left) and the NUHM1 (right) 1]. The vertical solid lines indicate the best-fit values, the horizontal solid lines are the $68 \%$ C.L. ranges, and the horizontal dashed lines are the $95 \%$ C.L. ranges for the indicated mass parameters.

nosity at $\sqrt{s}=14 \mathrm{TeV}$ 35, 36, and the proposed SLHC luminosity upgrade to $1000 / \mathrm{fb}$ of integrated luminosity at $\sqrt{s}=14 \mathrm{TeV}$ should permit the discovery of a gluino with $m_{\tilde{g}} \sim 3 \mathrm{TeV}$ 37 .

The central values of the masses of the supersymmetric partners of the $u, d, s, c, b$ quarks are slightly lighter than the gluino, as seen in Fig. 2. The difference between the gluino and the squark masses is sensitive primarily to $m_{0}$. The SUSY partners of the left-handed components of the four lightest quarks, the $\tilde{q}_{L}$, are predicted to be slightly heavier than the corresponding righthanded squarks, $\tilde{q}_{R}$, as seen by comparing the mass ranges in Fig. 2. As in the case of the gluino, squark masses up to $\sim 2.5 \mathrm{TeV}$ are allowed at the $3-\sigma$ level. Comparing the left and right panels, we see that the squarks are predicted to be somewhat lighter in the NUHM1 than in the CMSSM.

The scalar taus as well as the other scalar leptons are expected to be relatively light, as can be seen in Fig. 2. They would partially be in the reach of the ILC(500) (i.e. with $\sqrt{s}=500 \mathrm{GeV}$ ) and at the $95 \%$ C.L. nearly all be in the reach of the ILC(1000) [38, 39]. This also holds for the two lighter neutralinos and the light chargino.

\section{PREDICTIONS FOR THE LIGHTEST HIGGS MASS}

Finally we discuss the likelihood functions for $M_{h}$ within the CMSSM and NUHM1 frameworks obtained when dropping the contribution to $\chi^{2}$ from the direct Higgs searches at LEP. The results 

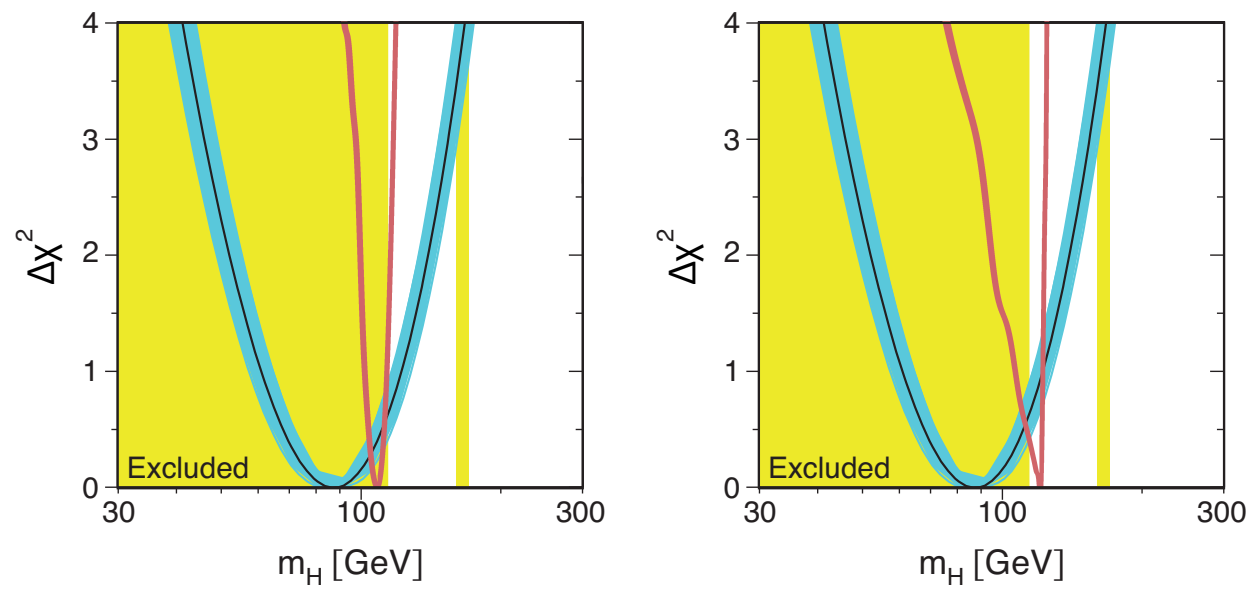

Figure 3. The $\chi^{2}$ functions for $M_{h}$ in the CMSSM (left) and the NUHM1 (right) 1], including the theoretical uncertainties (red bands). Overlayed is the blue band curve for the SM Higgs boson taken from Ref. [40]. Also shown is the mass range excluded for a SM-like Higgs boson (yellow shading). The LEP limits are not included in the fit.

are shown in the left and right panels of Fig. 3, respectively, together with the corresponding SM result 40 .

It is well known that the central value of the Higgs mass in a SM fit to the precision electroweak data lies below $100 \mathrm{GeV}$ 40, but the theoretical (blue bands in Fig. 3i) and experimental uncertainties in the SM fit are such that they are still compatible at the $95 \%$ C.L. with the direct lower limit of $114.4 \mathrm{GeV}$ 29] derived from searches at LEP. In the case of the CMSSM and NUHM1, one may predict $M_{h}$ on the basis of the underlying model parameters, with a 1- $\sigma$ uncertainty of $1.5 \mathrm{GeV}$ [17, shown as a red band in Fig. 3. Also shown in Fig. 3 are the LEP exclusion on a SM Higgs 29] and the Tevatron exclusion around $M_{H} \approx 165 \mathrm{GeV}$ [41] (yellow shading). The LEP exclusion is directly applicable to the CMSSM, since the $h$ couplings are essentially indistinguishable from those of the SM Higgs boson 30,31 , but this is not necessarily the case in the NUHM1, as discussed earlier.

In the case of the CMSSM, we see in the left panel of Fig. [3 that the minimum of the $\chi^{2}$ function occurs below the LEP exclusion limit. The fit result is still compatible at the $95 \%$ C.L. with the search limit, similarly to the SM case. As discussed above, a global fit including the LEP constraint has acceptable $\chi^{2}$. In the case of the NUHM1, shown in the right panel of Fig. 3, we see that the minimum of the $\chi^{2}$ function occurs above the LEP lower limit on the mass of a SM Higgs at $M_{h} \approx 121 \mathrm{GeV}$. Thus, within the NUHM1 the combination of all other experimental constraints naturally evades the LEP Higgs constraints, and no tension between $M_{h}$ and the experimental bounds exists.

\section{Acknowledgements}

S.H. thanks the organizers of L\&L 2010 for the invitation and the (as always!) inspiring atmosphere. We thank O. Buchmüller, R. Cavanaugh, A. De Roeck, J. Ellis, H. Flächer, G. Isidori, $\mathrm{K}$. Olive and F. Ronga with whom the results presented here have been obtained. Work supported in part by the European Community's Marie-Curie Research Training Network under contract MRTN-CT-2006-035505 'Tools and Precision Calculations for Physics Discoveries at Colliders' (HEPTOOLS).

\section{REFERENCES}

1. O. Buchmueller et al., Eur. Phys. J. C 64 (2009) 391.

2. O. Buchmueller et al., JHEP 0809 (2008) 117. 
3. LEP Supersymmetry Working Group, http://lepsusy.web.cern.ch/lepsusy/ .

4. M. Awramik, M. Czakon, A. Freitas and G. Weiglein, Phys. Rev. D 69 (2004) 053006.

5. S. Heinemeyer et al., JHEP 0608 (2006) 052.

6. M. Awramik, M. Czakon, A. Freitas and G. Weiglein, Phys. Rev. Lett. 93 (2004) 201805; M. Awramik, M. Czakon and A. Freitas, JHEP 0611 (2006) 048.

7. S. Heinemeyer, W. Hollik, A. Weber and G. Weiglein, JHEP 0804 (2008) 039.

8. M. Misiak et al., Phys. Rev. Lett. 98 (2007) 022002 .

9. M. Davier et al. Eur. Phys. J. C 66 (2010) 1.

10. Proceedings of the "Loops \& Legs" conferences, eds. J. Blümlein, T. Riemann et al., Nucl. Phys. B (Proc. Suppl.) 89 (2000); ibid. 116 (2003); ibid. 135 (2004); ibid. 160 (2006); ibid. 183 (2008).

11. O. Buchmueller et al., Phys. Lett. B 657 (2007) 87.

12. O. Buchmueller et al., Phys. Rev. D 81 (2010) 035009 .

13. See http://cern.ch/mastercode .

14. B. C. Allanach, Comput. Phys. Commun. 143 (2002) 305.

15. S. Heinemeyer, W. Hollik and G. Weiglein, Comput. Phys. Commun. 124 (2000) 76. See http: //www . feynhiggs.de .

16. S. Heinemeyer, W. Hollik and G. Weiglein, Eur. Phys. J. C 9 (1999) 343.

17. G. Degrassi et al., Eur. Phys. J. C 28 (2003) 133.

18. M. Frank et al., JHEP 0702 (2007) 047.

19. T. Moroi, Phys. Rev. D 53 (1996) 6565 [Erratum-ibid. D 56 (1997) 4424].

20. S. Heinemeyer, W. Hollik and G. Weiglein, Phys. Rept. 425 (2006) 265.

21. G. Isidori and P. Paradisi, Phys. Lett. B 639 (2006) 499.

22. G. Isidori, F. Mescia, P. Paradisi and D. Temes, Phys. Rev. D 75 (2007) 115019, and references therein.

23. F. Mahmoudi, Comput. Phys. Commun. 178 (2008) 745 .

24. D. Eriksson, F. Mahmoudi and O. Stal, JHEP 0811 (2008) 035.

25. G. Belanger, F. Boudjema, A. Pukhov and
A. Semenov, Comput. Phys. Commun. 176 (2007) 367; ibid. 149 (2002) 103; ibid. 174 (2006) 577.

26. P. Gondolo et al., New Astron. Rev. 49 (2005) 149.

27. P. Skands et al., JHEP 0407 (2004) 036.

28. B. Allanach et al., Comput. Phys. Commun. 180 (2009) 8.

29. R. Barate et al. [ALEPH, DELPHI, L3, OPAL Collaborations and LEP Working Group for Higgs boson searches], Phys. Lett. B 565 (2003) 61.

30. J. Ellis, S. Heinemeyer, K. Olive and G. Weiglein, Phys. Lett. B 515 (2001) 348.

31. S. Ambrosanio et al., Nucl. Phys. B 624 (2002) 3 .

32. P. Bechtle et al., Comput. Phys. Commun. 181 (2010) 138. See http://www.ippp.dur.ac.uk/HiggsBounds .

33. S. Schael et al. [ALEPH, DELPHI, L3, OPAL Collaborations and LEP Working Group for Higgs boson searches], Eur. Phys. J. C 47 (2006) 547.

34. CMS Note-2010/008.

35. G. Aad et al. [The ATLAS Collaboration], "Expected Performance of the ATLAS Experiment - Detector, Trigger and Physics," arXiv:0901.0512.

36. G. L. Bayatian et al., CMS Collaboration, CMS Technical Design Report, Volume II: Physics Performance, J. Phys. G 34, 995 (2007); see: http://cmsdoc.cern.ch/cms/cpt/tdr/ .

37. F. Gianotti et al., Eur. Phys. J. C 39 (2005) 293.

38. TESLA Technical Design Report [TESLA Collaboration], http://tesla.desy.de/ new_pages/TDR_CD/start.html .

39. G. Aarons et al. [ILC Collaboration], arXiv:0709.1893 [hep-ph].

40. LEP Electroweak Working Group, http://lepewwg.web.cern.ch/ LEPEWWG/Welcome.html .

41. T. Aaltonen et al. [CDF and D0 Collaborations], Phys. Rev. Lett. 104 (2010) 061802. 